\title{
Prediagnostic plasma branched-chain amino acids and the risk of amyotrophic lateral sclerosis
}

Kjetil Bjornevik, MD, PhD, Éilis J. O'Reilly, ScD, James D. Berry, MD, Clary B. Clish, PhD, Sarah Jeanfavre, MSc, Ikuko Kato, PhD, Laurence N. Kolonel, MD, PhD, Loic Le Marchand, MD, PhD, Marjorie L. McCullough, ScD, Sabrina Paganoni, MD, PhD, Michael A. Schwarzschild, MD, PhD, Evelyn O. Talbott, DrPH, Robert B. Wallace, MD, Zhongli Zhang, PhD, JoAnn E. Manson, MD, DrPH, and Alberto Ascherio, MD, DrPH Neurology ${ }^{\circledR}$ 2019;92:e2081-e2088. doi:10.1212/WNL.0000000000006669

\section{Abstract \\ Objective}

To assess whether prediagnostic levels of plasma branched-chain amino acids (BCAAs) are associated with amyotrophic lateral sclerosis (ALS) risk.

\section{Methods}

We included participants from 5 large cohort studies-The Nurses' Health Study, the Health Professionals Follow-up Study, the Cancer Prevention Study II Nutrition, the Multiethnic Cohort Study, and the Women's Health Initiative-and identified 275 individuals who developed ALS during follow-up. Two controls were randomly selected for each case, matched on cohort, age, sex, fasting status, and time of blood draw. We measured metabolites using liquid chromatography-mass spectrometry and used conditional logistic regression to estimate rate ratios (RRs) and 95\% confidence intervals (CIs) for the association of individual BCAAs with ALS risk.

\section{Results}

None of the 3 BCAAs was associated with a higher ALS risk. The risk estimates were similar for leucine ( $R R$ top vs bottom quartile: $0.87,95 \% \mathrm{CI} 0.57-1.33$ ), isoleucine (RR top vs bottom quartile: $0.81,95 \%$ CI $0.52-1.24$ ), and valine (RR top vs bottom quartile: $0.80,95 \% \mathrm{CI}$ $0.52-1.23$ ) in a multivariable analysis adjusted for body mass index, smoking, level of education, and physical activity. The estimates did not vary significantly by sex, fasting status, or time interval between blood draw and disease onset.

\section{Conclusion}

The results from this study do not support the hypothesis that BCAAs are risk factors for ALS.

\author{
Correspondence \\ Dr. Bjornevik \\ kbjorne@hsph.harvard.edu
}




\section{Glossary}

ALS = amyotrophic lateral sclerosis; BCAA = branched-chain amino acids; $\mathbf{B M I}=$ body mass index; $\mathbf{C I}=$ confidence interval; CPS-II Nutrition = Cancer Prevention Study II Nutrition Cohort; HPFS = Health Professionals Follow-up Study; ICD-9 = International Classification of Diseases, Ninth Revision; MEC = Multiethnic Cohort Study; NHS = Nurses' Health Study; $\mathbf{N M R}=$ nuclear magnetic resonance; $\mathbf{O R}=$ odds ratio; $\mathbf{P P V}=$ positive predictive value; $\mathbf{R R}=$ rate ratio; $\mathbf{W H I}=$ Women's Health Initiative.

The branched-chain amino acids (BCAAs) valine, leucine, and isoleucine play an important role in glutamate metabolism, ${ }^{1}$ which has been implicated in the pathogenesis of amyotrophic lateral sclerosis (ALS). ${ }^{2}$ As BCAAs have been postulated to restore impaired glutamate metabolism, ${ }^{3}$ the effect of these essential amino acids on ALS progression has been explored in clinical trials. Several of these have, however, not only failed to support a beneficial role of BCAAs but provided evidence for potential adverse effects. In an Italian trial, there was an excess of deaths in the group treated with BCAAs, ${ }^{4}$ and in a later US trial, the estimated decline in forced vital capacity, one of the a priori selected outcomes, was 2.5 times greater in the treatment group as compared to placebo. ${ }^{5}$ As BCAAs may affect pathways relevant to ALS, including downregulation of antioxidant genes associated with familial ALS and induction of hyperexcitability in cortical neurons, ${ }^{6,7}$ it has been proposed that dietary supplements containing BCAAs, which are commonly used by Italian soccer players, ${ }^{8}$ could be one of the factors that contributed to their elevated ALS risk..$^{9,10}$

Whether BCAAs increase the risk of ALS is currently not known. Higher levels have been reported in patients with ALS compared to controls, ${ }^{11,12}$ but as the amino acids' concentrations may have been affected by the disease itself, it is unclear whether they are etiologically relevant. Prospective studies including prediagnostic samples from individuals later developing ALS could be more informative, but they are difficult to conduct due to the low incidence of the disease.

To address this, we conducted a pooled analysis of 5 large prospective cohort studies to assess whether higher prediagnostic plasma levels of BCAAs are associated with an increased ALS risk.

\section{Methods}

\section{Study populations}

The study population included participants from 5 large cohort studies: The Nurses' Health Study (NHS), the Health Professionals Follow-up Study (HPFS), the Cancer Prevention Study II Nutrition Cohort (CPS-II Nutrition), the Multiethnic Cohort Study (MEC), and the Women's Health Initiative (WHI). Detailed descriptions of the individual cohorts have previously been published. ${ }^{13-17}$ In short, the NHS began in 1976 with 121,700 female nurses aged 30-55 years at baseline. ${ }^{13}$ In 1988, all nurses were invited to provide a blood sample, which was collected from 32,826 women during 1989 and 1990. Thirty-nine nurses who gave a blood sample developed ALS between blood draw and end of follow-up. The HPFS began in 1986 and enrolled 51,529 male health professionals aged $40-75$ years at baseline. ${ }^{14}$ All of the participants were invited to provide a blood sample, which was collected from 18,018 men between 1993 and 1995. Twenty-six men were identified with ALS between blood draw and end of follow-up. Every 2 years, the participants in these 2 cohorts were followed up with similar questionnaires on medical history and health-related behavior. The CPS-II Nutrition cohort was established in 1992 and consists of a subpopulation of the larger CPS-II cohort. ${ }^{15}$ It includes 86,406 men and 97,788 women aged $50-74$ years at baseline. These participants completed a questionnaire on lifestyle and dietary factors at baseline, in 1997 and biennially thereafter. In 1998, participants living in urban and suburban areas were invited to provide a blood sample, which was collected from 39,380 members of the cohort. Among these, 58 participants died of ALS during follow-up. The MEC cohort study began in 1993 and consists of 96,810 men and 118,441 women aged 45-75 years at baseline with the self-reported ethnic background of African American, Japanese American, Latino, Native Hawaiian, or white. ${ }^{16}$ The participants completed a lifestyle and disease history questionnaire at baseline, and follow-up questionnaires every 5 years thereafter. A total of 67,594 members of the cohort provided a blood sample, which was largely collected between 2001 and 2006. Thirtyone participants died of ALS between blood draw and end of follow-up. The WHI study recruited a total of 161,809 postmenopausal women aged 50-79 years between 1993 and 1998 , who were enrolled into one or more of 3 clinical trials ( $\mathrm{n}$ $=68,133)$ or into the observational study $(\mathrm{n}=93,676) .{ }^{17} \mathrm{At}$ baseline, all of the participants completed a questionnaire on lifestyle and disease history and provided a blood sample. A total of 121 participants died of ALS after blood draw until end of follow-up. End of follow-up was December 31, 2010, for NHS, HPFS, and CPS-II Nutrition; September 30, 2012, for WHI; and December 31, 2012, for MEC.

\section{Standard protocol approvals, registrations, and patient consents}

All of the studies included were reviewed and approved by the institutional review board at the institution where each study was conducted.

\section{Endpoint definition}

In the CPS-II Nutrition, MEC, and WHI studies, we identified ALS cases through a search of the National Death Index. WHI 
also compiled self-reported ALS information for women in the observational study, but it was never adjudicated. All individuals with ICD-9 code 335.2 (motor neuron disease) listed as the underlying or contributing cause of death were considered to have had ALS. In a previous validation study, ALS was the primary diagnosis in $90 \%$ of the individuals for whom code 335.2 was listed as the cause or contributory cause of death. ${ }^{18}$ We assigned the date of onset to 3 years before the date of death, based on median survival among patients with ALS. ${ }^{19}$

In the NHS and HFPS, we identified incident ALS by selfreport on the biennial questionnaire. For participants reporting a diagnosis of ALS, we requested permission to contact the treating neurologist and obtain a copy of the medical records. Due to the progressive nature of ALS, many of the patients had already died before we could obtain permission to release the medical records. For these patients, we sent the request to the closest family member. We asked the treating neurologist to complete a questionnaire, which was based on the revised El Escorial criteria, on the certainty of the diagnosis (definite, probable, or possible), and the clinical history. The final confirmation of the diagnosis was made after reviewing medical records by a neurologist with experience in ALS diagnosis. We observed few discrepancies between the diagnosis made by the field neurologist and the neurologist with expertise in ALS, and the differences were mostly limited to whether patients were diagnosed with definite or probable ALS. Patients defined as definite or probable cases were included in the study. If we were unable to obtain a copy of the medical record or the neurologist's questionnaire to confirm the diagnosis of self-reported ALS, only the patients with ALS specifically listed on the death certificate were included.

For each ALS case, we randomly selected 2 controls who were alive at the time of the case diagnosis and matched on cohort, birth year ( \pm 1 year), sex, ethnicity, fasting status, and time of blood draw. For one of the cases, only one matched control was included in the final analyses, as the assessment of metabolites failed in the other matched control.

\section{Assessment of metabolites}

Across the cohorts, blood samples were collected from a total of 319,627 participants and were stored at $-70^{\circ} \mathrm{C}$ or below. For the individuals who developed ALS and their matched controls, $10 \mu \mathrm{L}$ of plasma from each individual was used for the metabolomics analysis. Triplets of plasma samples (one case with its 2 matched controls) were handled identically and assayed in the same analytic run. The order of the plasma samples within each case-control tripled was arranged at random, to ensure that all assays were conducted without knowledge of the case/control status. We profiled the metabolites using hydrophilic interaction liquid chromatography coupled to positive ion mode mass spectrometry at the Broad Institute (Boston, MA), as previously described. $^{20}$

\section{Assessment of covariates}

Information on other covariates of interest, including smoking status, height, weight, educational level, physical activity, and diabetes status, was collected at baseline for all cohorts, and every 2 years since baseline in HPFS and NHS. Participants self-reported a diagnosis of type 1 or type 2 diabetes mellitus. These specific covariates were included in multivariable models, as they have previously been associated with ALS risk, and could be potential confounders. The nearest covariate data collected before or at the time of blood draw were used.

\section{Statistical analysis}

To account for the matched study design, we used conditional logistic regression to estimate odds ratios (ORs) and 95\% confidence intervals (CIs) for the association of BCAAs with ALS risk. As controls in our study were selected using risk-set sampling, the ORs estimate incidence rate ratios (RRs). ${ }^{21}$ We log-transformed and standardized the amino acids within cohort and sex to account for possible differences that may have been introduced as cohort samples were processed at different times. We estimated the correlation between log-transformed levels of individual BCAAs, glutamate, urate, and body mass index (BMI) using Pearson correlation, and tested whether the BCAAs were significantly different among diabetic and nondiabetic participants using a 2 -sided $t$ test. The amino acids were modeled as both continuous (per 1 SD increase) and categorical variables to maximize power and to explore possible nonlinear relationships. For the categorical analyses, we categorized the participants into cohort and sex-specific quartiles based on the distribution among the controls. We tested for a linear trend across the quartiles by assigning the median value to each quartile and modeled this as a continuous variable. Multivariable models were used to assess the influence of possible confounders, including BMI (using weight reported closest to time of blood draw, continuous), educational level ( $<$ high school, high school, $>$ high school), smoking status (never smoker, past smoker, current smoker), and physical activity (approximate tertiles corresponding to low, medium, and high activity levels). We categorized participants according to tertiles of metabolic equivalent hours of total physical activity in NHS, HPFS, and WHI, while the amount of moderate and vigorous physical activity was used for CPSII and MEC. When data were missing for a covariate, we used a missing indicator $(6.7 \%$ of the participants had 1 or more missing covariate data). We tested for effect modification by sex, fasting status ( $<4$ vs $\geq 4$ hours since last meal), and time from blood collection to disease onset ( $<5$ vs $\geq 5$ years) by including a product term of individual BCAAs and the modifier in separate models. We conducted a sensitivity analysis excluding individuals with diabetes, as diabetes has been associated with marked alterations in the metabolome, ${ }^{22}$ including elevated BCAA levels, ${ }^{23}$ and has also been associated with the risk of ALS. ${ }^{24}$ All of the analyses were conducted using SAS 9.4 (SAS Institute, Cary, NC). The $\alpha$-level was set at 0.05 . 


\section{Data availability}

The datasets analyzed in the current study are not publicly available because of restricted access, but further information about the datasets is available from the corresponding author on reasonable request.

\section{Results}

We identified a total of 275 individuals who developed ALS among the 319,627 participants who provided blood samples in these 5 cohort studies. There was a higher proportion of female compared to male participants in our study. The cases were slightly more likely to have ever smoked compared to controls and had slightly lower BMI at baseline (table 1). Further, fewer cases had higher educational level than high school compared to the controls. The other characteristics were similarly distributed. Plasma levels of leucine, isoleucine, and valine correlated significantly with each other, and higher levels correlated with higher BMI (table 2). Further, higher levels of the amino acids correlated significantly with higher levels of glutamate and with urate. The levels of all 3 BCAAs were higher among diabetic compared to nondiabetic participants ( $p=0.006, p=0.004$, and $p<0.001$ by the $t$ test for leucine, isoleucine, and valine, respectively).

Higher levels of valine were associated with a lower ALS risk in analyses adjusted for age, sex, and matching variables, but the trend was not monotonic and was no longer statistically significant after further adjustment for BMI. Other BCAAs were not significantly associated with ALS risk, neither in the continuous nor in the categorical analyses (table 3). Adjustment for BMI, smoking, physical activity, and educational level did not materially alter the results. Total BCAA plasma level was not associated with ALS risk (data not shown).

None of the 3 metabolites was significantly associated with ALS risk in any of the predefined subgroup analyses (table 4). The RR estimates were slightly lower in women compared to men, but the difference was not significant. Similarly, the estimates were lower among those with fasting samples compared to those with nonfasting samples, but the estimates were not statistically significantly different. The estimates from analyses restricted to those with longer interval $(\geq 5$ years) between blood draw and ALS onset were similar to those restricted to those with shorter interval ( $<5$ years). The results were similar when we restricted the analyses to individuals with an interval of 2 years or less between blood draw and ALS onset (data not shown).

In a sensitivity analysis, we excluded participants who had developed diabetes before the blood draw, as insulin resistance has been associated with increased BCAA levels and diabetes has been suggested to affect ALS risk. ${ }^{23,24}$ However, none of the 3 BCAAs was associated with ALS risk when excluding patients with diabetes $(\mathrm{n}=49)$. In a multivariable model adjusting for age, sex, BMI, smoking, physical activity, and educational level, the RR for ALS per 1 SD increase in amino acid levels was 0.88 (95\% CI 0.76-1.03) for leucine, 0.92 (95\% CI $0.78-1.08$ ) for isoleucine, and 0.87 (95\% CI 0.75-1.02) for valine.

\section{Discussion}

In this pooled analysis of 5 large cohort studies, we did not find any association between prediagnostic plasma levels of BCAAs and the risk of ALS. The results did not vary significantly with time to ALS onset and were not materially changed when we adjusted for the most relevant confounders. Our findings do not support the hypothesis that plasma BCAAs are risk factors for ALS.

Previous research on BCAAs and ALS risk is sparse, and no study has previously evaluated the association between prediagnostic plasma levels and disease risk. Some, ${ }^{11,12,25}$ but not all, ${ }^{26,27}$ studies comparing the metabolome in patients with ALS with controls reported alterations in the metabolism of BCAAs in patients. In a study comparing the metabolome in 22 patients with ALS with 28 controls with other neurologic conditions, the plasma levels of all 3 BCAAs were higher in patients with ALS compared to controls in analyses using nuclear magnetic resonance (NMR) spectroscopy. ${ }^{11}$ The results were similar in a study comparing the metabolome in the same cohort of patients and controls using mass spectrometry, but only plasma levels of valine and isoleucine were significantly increased in patients with ALS. ${ }^{12}$ Further, one study used NMR to try to identify a diagnostic profile discriminating 95 patients with motor neuron disease from 86 patients with other neurologic diseases and reported that valine and isoleucine measured in the CSF were among the metabolites contributing most to the final diagnostic model. ${ }^{25}$ In our study, we did not find any differences in prediagnostic BCAA levels in those later developing ALS compared to their matched controls, which indicates that the amino acids do not contribute to the earliest etiologic events. Still, it is possible that these amino acids affect disease processes at a later stage, which could be consistent with the results from previous metabolomics studies, the possible adverse effects reported in clinical trials, ${ }^{4,5}$ and the findings from in vivo and in vitro studies suggesting that BCAAs affect pathways relevant to the disease. ${ }^{6,7}$

BCAAs contribute both to de novo synthesis and buffering of glutamate, which in excessive amounts may contribute to excitotoxicity in ALS. The amino acids, in particular leucine, rapidly cross into the $\mathrm{CNS},{ }^{28}$ and provide approximately $25 \%$ of the amino groups of brain glutamate. ${ }^{29}$ It has been suggested that high levels of BCAAs lead to an excess of glutamate, which could trigger motor neuron death due to excessive stimulation of glutamate receptors. ${ }^{30}$ This is supported by evidence from an animal study where a diet enriched in BCAAs induced a hyperexcitability of motor neurons, which was related to a specific glutamate receptor (NMDA receptor), as the effect was abolished after 
Table 1 Selected age-standardized characteristics

\begin{tabular}{|c|c|c|c|}
\hline Characteristics & Cases $(n=275)$ & Controls $(n=549)$ & $p$ Value \\
\hline \multicolumn{4}{|l|}{ Age, $y^{a}$} \\
\hline Mean (SD) & $64.6(7.2)$ & $64.6(7.2)$ & - \\
\hline \multicolumn{4}{|l|}{ Sex, $n(\%)^{a}$} \\
\hline Male & 75 (27.3) & $150(27.3)$ & - \\
\hline Female & $200(72.7)$ & 399 (72.7) & \\
\hline \multicolumn{4}{|l|}{ Fasting status, $\mathrm{h}, \mathrm{n}(\%)^{\mathrm{a}}$} \\
\hline$<4$ & 73 (26.6) & $155(28.2)$ & - \\
\hline$\geq 4$ & $202(73.5)$ & $394(71.8)$ & \\
\hline \multicolumn{4}{|c|}{ Interval from blood draw to ALS onset, $y^{a}$} \\
\hline Median (IQR) & $4.8(1.9-7.8)$ & - & - \\
\hline$<5, \mathrm{n}(\%)$ & $144(52.4)$ & - & - \\
\hline$\geq 5, n(\%)$ & $131(47.6)$ & - & - \\
\hline \multicolumn{4}{|l|}{ Body mass index } \\
\hline Mean (SD) & $26.2(4.4)$ & $26.9(5.2)$ & 0.034 \\
\hline Smoking status, $\mathbf{n}(\%)^{a}$ & & & 0.82 \\
\hline Never smoker & $123(44.7)$ & $264(48.1)$ & \\
\hline Past smoker & $128(46.6)$ & $239(43.5)$ & \\
\hline Current smoker & $18(6.6)$ & $36(6.6)$ & \\
\hline Unknown & $6(2.2)$ & $10(1.8)$ & \\
\hline Ethnicity, n (\%) & & & 0.98 \\
\hline White & $225(81.8)$ & $448(81.6)$ & \\
\hline African American & $21(7.6)$ & $39(7.1)$ & \\
\hline Hispanic & $8(2.9)$ & $17(3.1)$ & \\
\hline Asian & $10(3.6)$ & $20(3.6)$ & \\
\hline Other & $6(2.2)$ & $17(3.1)$ & \\
\hline Not reported & $5(1.8)$ & $8(1.5)$ & \\
\hline Education, $\mathrm{n}(\%)^{\mathrm{a}}$ & & & 0.50 \\
\hline$<$ High school & $13(4.8)$ & $20(3.7)$ & \\
\hline High school & $48(17.7)$ & $84(15.4)$ & \\
\hline > High school & $211(77.6)$ & $442(81.0)$ & \\
\hline Physical activity, $\mathrm{n}(\%)^{\mathrm{a}}$ & & & 0.99 \\
\hline Low levels & $87(32.8)$ & $175(33.0)$ & \\
\hline Medium level & 79 (29.8) & 157 (29.6) & \\
\hline High level & 99 (37.4) & 199 (37.5) & \\
\hline Diabetes, n (\%) & & & 0.46 \\
\hline Yes & $14(5.1)$ & $35(6.4)$ & \\
\hline No & $261(94.9)$ & $514(93.6)$ & \\
\hline
\end{tabular}

Abbreviation: IQR = interquartile range.

Values are standardized to the age distribution of the study population. Values of polytomous variables may not sum up to $100 \%$ due to rounding. $p$ Values are not given for matching factors (age, sex, and fasting status), as these are similar in the 2 groups by design.

a Value is not age-adjusted. 
Table 2 Pearson correlation coefficients for individual branched-chain amino acids, urate, and body mass index (BMI)

\begin{tabular}{lllll}
\hline & Leucine & Isoleucine & Valine & Glutamate \\
\hline Isoleucine & $0.936^{\mathrm{a}}$ & & & \\
\hline Valine & $0.894^{\mathrm{a}}$ & $0.862^{\mathrm{a}}$ & & \\
\hline Glutamate & $0.333^{\mathrm{a}}$ & $0.317^{\mathrm{a}}$ & $0.319^{\mathrm{a}}$ & $0.197^{\mathrm{a}}$ \\
\hline Urate & $0.158^{\mathrm{a}}$ & $0.169^{\mathrm{a}}$ & $0.188^{\mathrm{a}}$ & $0.227^{\mathrm{a}}$ \\
\hline BMI & $0.206^{\mathrm{a}}$ & $0.224^{\mathrm{a}}$ & $0.272^{\mathrm{a}}$ & $0.302^{\mathrm{a}}$ \\
\hline
\end{tabular}

${ }^{a} p<0.001$ (2-tailed).

administration of a specific NMDA channel blocker. ${ }^{31}$ However, BCAAs also serve as a buffering mechanism against excessive glutamate levels. After BCAAs are metabolized in astrocytes, their ketoacids are released into the extracellular fluid and can subsequently be taken up by neurons. ${ }^{1}$ Ketoleucine can be converted back to leucine in a process that consumes glutamate, which provides a mechanism for preventing excessive amounts of the amino acid. ${ }^{1}$ It is thus unclear whether higher BCAA intake in humans would lead to levels of glutamate that could have neurotoxic effects.
Our study has several strengths. First, the nested case-control study design, where the participants were drawn from existing cohort studies, minimizes the risk of selection bias when choosing the controls. Second, the prospective study design with samples collected years before disease onset limits the risk of reverse causation bias. Finally, we were able to account for several possible confounders suggested to be relevant in ALS, including BMI, physical activity, smoking, and diabetes.

Our study also has some limitations. While we drew participants from 5 large cohort studies, we were able to identify

Table 3 Rate ratio (RR) for amyotrophic lateral sclerosis according to prediagnostic branched amino acid plasma levels

\begin{tabular}{|c|c|c|c|}
\hline \multirow[b]{2}{*}{ Incident cases (275 cases, 549 noncases) } & \multicolumn{3}{|l|}{ RR (95\% Cl) } \\
\hline & Leucine & Isoleucine & Valine \\
\hline \multicolumn{4}{|l|}{ Model $1^{a}$} \\
\hline Per SD & $0.88(0.76-1.02)$ & $0.90(0.78-1.04)$ & $0.86(0.75-0.99)$ \\
\hline \multicolumn{4}{|l|}{ Across quartiles } \\
\hline Q1 & 1 (reference) & 1 (reference) & 1 (reference) \\
\hline Q2 & $0.81(0.54-1.23)$ & $0.77(0.51-1.15)$ & $0.78(0.52-1.16)$ \\
\hline Q3 & $0.67(0.44-1.01)$ & $0.72(0.48-1.07)$ & $0.51(0.33-0.78)$ \\
\hline Q4 & $0.81(0.54-1.23)$ & $0.74(0.49-1.11)$ & $0.72(0.48-1.09)$ \\
\hline$p$ For trend & 0.23 & 0.13 & 0.046 \\
\hline \multicolumn{4}{|l|}{ Model $2^{b}$} \\
\hline Per SD & $0.90(0.78-1.05)$ & $0.93(0.80-1.09)$ & $0.89(0.77-1.04)$ \\
\hline \multicolumn{4}{|l|}{ Across quartiles } \\
\hline Q1 & 1 (reference) & 1 (reference) & 1 (reference) \\
\hline Q2 & $0.85(0.56-1.29)$ & $0.80(0.53-1.21)$ & $0.80(0.53-1.20)$ \\
\hline Q3 & $0.72(0.47-1.09)$ & $0.77(0.51-1.16)$ & $0.54(0.35-0.83)$ \\
\hline Q4 & $0.87(0.57-1.33)$ & $0.81(0.52-1.24)$ & $0.80(0.52-1.23)$ \\
\hline$p$ For trend & 0.40 & 0.29 & 0.14 \\
\hline
\end{tabular}

Abbreviation: $\mathrm{Cl}=$ confidence interval.

${ }^{a}$ Model 1: adjusted for age, sex, and matching variables.

${ }^{\mathrm{b}}$ Model 2: additional adjustment for body mass index, smoking, level of education, and physical activity. 
Table 4 Rate ratio (RR) for amyotrophic lateral sclerosis (ALS) according to prediagnostic branched amino acid plasma levels in specific subgroups

\begin{tabular}{|c|c|c|c|}
\hline \multirow[b]{2}{*}{ Subgroup } & \multicolumn{3}{|c|}{ RR per 1 SD increase $(95 \% \mathrm{Cl})^{a}$} \\
\hline & Leucine & Isoleucine & Valine \\
\hline \multicolumn{4}{|l|}{ Sex } \\
\hline Female & $0.87(0.72-1.04)$ & $0.87(0.72-1.05)$ & $0.87(0.73-1.05$ \\
\hline Male & $0.96(0.72-1.28)$ & $1.04(0.78-1.38)$ & $0.92(0.67-1.25$ \\
\hline$p$ For interaction & 0.35 & 0.15 & 0.64 \\
\hline \multicolumn{4}{|l|}{ Fasting status, $\mathrm{h}$} \\
\hline$<4$ & $1.15(0.82-1.59)$ & $1.12(0.80-1.56)$ & $1.14(0.83-1.57$ \\
\hline$\geq 4$ & $0.83(0.69-1.01)$ & $0.88(0.72-1.07)$ & $0.85(0.70-1.03$ \\
\hline$p$ For interaction & 0.10 & 0.23 & 0.14 \\
\hline \multicolumn{4}{|c|}{ Interval between blood draw and ALS onset, y } \\
\hline$<5$ & $0.92(0.75-1.13)$ & $1.01(0.81-1.25)$ & $0.83(0.67-1.04$ \\
\hline$\geq 5$ & $0.87(0.70-1.09)$ & $0.86(0.69-1.08)$ & $0.93(0.75-1.16$ \\
\hline$p$ For interaction & 0.69 & 0.28 & 0.61 \\
\hline
\end{tabular}

Abbreviation: $\mathrm{Cl}=$ confidence interval.

a Adjusted for age, sex, body mass index, smoking, level of education, physical activity, and matching factors.

only 275 individuals who later developed ALS, due to the low incidence of the disease. This may have affected the statistical power to detect significant differences, especially in subgroup analyses. Still, given that risk estimates for all of the BCAAs with ALS risk were below 1.0, it seems unlikely that the amino acids would be associated with an increased risk if a higher number of participants had been included. Our study population only included $27 \%$ male participants, which represents an atypical ALS population, as the incidence rate of the disease is higher in men compared to women. ${ }^{32}$ The unusual distribution is mainly due to the inclusion of participants from WHI, who are all female and all contributed with blood samples. We did not observe any significant differences in the results in men and women, and it is therefore unlikely that this has affected our results. We did not have information on clinical covariates, such as subtypes of ALS, and could therefore not evaluate whether there were differences between those developing familial ALS to those with sporadic ALS. Further, due to lack of clinical information, we used information from death certificates to capture ALS cases in CPS-IIN, MEC, and WHI. This may have led to misclassification of ALS in some participants, as death certificates are likely to be less accurate than medical records. In a US study, the sensitivity and positive predictive value (PPV) for use of death certificates for ALS were estimated to be $85 \%$ and $65 \%,{ }^{33}$ while a recent study from Denmark reported a sensitivity of $84 \%$ and an overall PPV of $82 \% .{ }^{34}$ Overall, this suggests that use of death certificates is an adequate, but not ideal, tool to capture patients with ALS in epidemiologic studies. We assessed the association using a single plasma measurement per individual, which may not reflect long-term exposure of the amino acids over time. However, in a validation study in NHS and HPFS, the 3 amino acids had Spearman correlations above 0.60 when comparing 2 samples collected in the same individuals $0.8-2.3$ years apart, indicating that a single measurement may reasonably reflect longer-term levels. ${ }^{35}$ As data on physical activity were collected at different ages and with different methodologies across the cohorts, we may not have been able to fully adjust for this in the analyses. Finally, as is inherent to any observational study, we cannot exclude the possibility that the results may be affected by residual or unmeasured confounding that we cannot account for.

In a pooled analysis of 5 cohort studies, we did not find any significant association between prediagnostic plasma levels of BCAAs and ALS risk. Our findings suggest that high physiologic levels of these amino acids do not increase the risk of the disease.

\section{Author contributions}

Dr. Bjornevik: drafting/revising the manuscript for content, analysis or interpretation of data, statistical analysis. Dr. O'Reilly: drafting/revising the manuscript for content, analysis or interpretation of data. Dr. Berry: drafting/revising the manuscript for content, analysis or interpretation of data. Dr. Clish: drafting/revising the manuscript for content, analysis or interpretation of data. S. Jeanfavre: drafting/revising the manuscript for content, analysis or interpretation of data. Dr. Kato: drafting/revising the manuscript for content, 
acquisition of data. Dr. Kolonel: drafting/revising the manuscript for content, acquisition of data. Dr. Le Marchand: drafting/revising the manuscript for content, acquisition of data. Dr. McCullough: drafting/revising the manuscript for content, acquisition of data. Dr. Paganoni: drafting/revising the manuscript for content, analysis or interpretation of data. Dr. Schwarzschild: drafting/revising the manuscript for content, analysis or interpretation of data. Dr. Talbott: drafting/ revising the manuscript for content, acquisition of data. Robert B. Wallace: drafting/revising the manuscript for content, acquisition of data. Dr. Zhang: drafting/revising the manuscript for content, analysis or interpretation of data. Dr. Manson: drafting/revising the manuscript for content, acquisition of data. Dr. Ascherio: study concept or design, drafting/revising the manuscript for content, analysis or interpretation of data, acquisition of data, obtaining funding. Drs. Bjornevik and Ascherio had full access to all the data in the study and take responsibility for the integrity of the data and the accuracy of the data analysis.

\section{Acknowledgment}

The authors acknowledge the cohorts' participants and Leslie Unger for administrative support.

\section{Study funding}

This work was supported by a grant from the National Institute of Neurologic Diseases and Stroke (R01 NS045893) awarded to Alberto Ascherio. The NHS is funded by the NIH through grants UM1 CA186107 and R01 CA49449. The HPFS cohort is funded by the NIH through grant UM1 CA167552. The American Cancer Society funds the creation, maintenance, and updating of the CPS-II cohorts. The MEC cohort is funded by the NIH through U01 CA164973. The WHI program is funded by the National Heart, Lung, and Blood Institute, NIH, US Department of Health and Human Services, through contracts HHSN268201600018C, HHSN268201600001C, and HHSN268201600002C. The sponsors had no role in the design and conduct of the study; collection, management, analysis, and interpretation of the data; or preparation, review, or approval of the manuscript.

\section{Disclosure}

The authors report no disclosures relevant to the manuscript. Go to Neurology.org/ $\mathrm{N}$ for full disclosures.

\section{Publication history}

Received by Neurology April 6, 2018. Accepted in final form September 17, 2018.

\section{References}

1. Yudkoff M, Daikhin Y, Nissim I, et al. Brain amino acid requirements and toxicity: the example of leucine. J Nutr 2005; 135:1531S-1538S.

2. Plaitakis A. Glutamate dysfunction and selective motor neuron degeneration in amyotrophic lateral sclerosis: a hypothesis. Ann Neurol 1990;28:3-8.

3. Plaitakis A, Smith J, Mandeli J, Yahr MD. Pilot trial of branched-chain aminoacids in amyotrophic lateral sclerosis. Lancet 1988;1:1015-1018.

4. The Italian ALS Study Group. Branched-chain amino acids and amyotrophic lateral sclerosis: a treatment failure? Neurology 1993;43:2466-2470.
5. Tandan R, Bromberg MB, Forshew D, et al. A controlled trial of amino acid therapy in amyotrophic lateral sclerosis: I: clinical, functional, and maximum isometric torque data. Neurology 1996;47:1220-1226.

6. Piscopo P, Crestini A, Adduci A, et al. Altered oxidative stress profile in the cortex of mice fed an enriched branched-chain amino acids diet: possible link with amyotrophic lateral sclerosis? J Neurosci Res 2011;89:1276-1283.

7. Carunchio I, Curcio L, Pieri M, et al. Increased levels of p70S6 phosphorylation in the G93A mouse model of amyotrophic lateral sclerosis and in valine-exposed cortical neurons in culture. Exp Neurol 2010;226:218-230.

8. Taioli E. Use of permitted drugs in Italian professional soccer players. Br J Sports Med 2007;41:439-441.

9. Chio A, Benzi G, Dossena M, Mutani R, Mora G. Severely increased risk of amyotrophic lateral sclerosis among Italian professional football players. Brain 2005;128: 472-476.

10. Chio A, Calvo A, Dossena M, Ghiglione P, Mutani R, Mora G. ALS in Italian professional soccer players: the risk is still present and could be soccer-specific. Amyotroph Lateral Scler 2009; 10:205-209.

11. Wu JF, Wuolikainen A, Trupp M, et al. NMR analysis of the CSF and plasma metabolome of rigorously matched amyotrophic lateral sclerosis, Parkinson's disease and control subjects. Metabolomics 2016;12.

12. Wuolikainen A, Jonsson P, Ahnlund M, et al. Multi-platform mass spectrometry analysis of the CSF and plasma metabolomes of rigorously matched amyotrophic lateral sclerosis, Parkinson's disease and control subjects. Mol Biosyst 2016;12:1287-1298.

13. Bao Y, Bertoia ML, Lenart EB, et al. Origin, methods, and evolution of the three nurses' health studies. Am J Public Health 2016;106:1573-1581.

14. Rimm EB, Giovannucci EL, Willett WC, et al. Prospective study of alcohol con sumption and risk of coronary disease in men. Lancet 1991;338:464-468.

15. Calle EE, Rodriguez C, Jacobs EJ, et al. The American Cancer Society Cancer Prevention Study II Nutrition cohort: rationale, study design, and baseline characteristics. Cancer 2002;94:2490-2501.

16. Kolonel LN, Henderson BE, Hankin JH, et al. A multiethnic cohort in Hawaii and Los Angeles: baseline characteristics. Am J Epidemiol 2000;151:346-357.

17. Anderson GL, Manson J, Wallace R, et al. Implementation of the women's health initiative study design. Ann Epidemiol 2003;13:S5-S17.

18. Weisskopf MG, McCullough ML, Calle EE, Thun MJ, Cudkowicz M, Ascherio A. Prospective study of cigarette smoking and amyotrophic lateral sclerosis. Am J Epidemiol 2004;160:26-33.

19. Traxinger K, Kelly C, Johnson BA, Lyles RH, Glass JD. Prognosis and epidemiology of amyotrophic lateral sclerosis: analysis of a clinic population, 1997-2011. Neurol Clin Pract 2013;3:313-320.

20. Paynter NP, Balasubramanian R, Giulianini F, et al. Metabolic predictors of incident coronary Heart disease in women. Circulation 2018;137:841-853.

21. Knol MJ, Vandenbroucke JP, Scott P, Egger M. What do case-control studies estimate? Survey of methods and assumptions in published case-control research. Am J Epidemiol 2008; 168:1073-1081.

22. Guasch-Ferre M, Hruby A, Toledo E, et al. Metabolomics in prediabetes and diabetes: a systematic review and meta-analysis. Diabetes Care 2016;39:833-846.

23. Mahendran Y, Jonsson A, Have CT, et al. Genetic evidence of a causal effect of insulin resistance on branched-chain amino acid levels. Diabetologia 2017;60:873-878.

24. Kioumourtzoglou MA, Rotem RS, Seals RM, Gredal O, Hansen J, Weisskopf MG. Diabetes mellitus, obesity, and diagnosis of amyotrophic lateral sclerosis: a population-based study. JAMA Neurol 2015;72:905-911.

25. Blasco H, Nadal-Desbarats L, Pradat PF, et al. Untargeted $1 \mathrm{H}-\mathrm{NMR}$ metabolomics in CSF: toward a diagnostic biomarker for motor neuron disease. Neurology 2014;82: $1167-1174$.

26. Lawton KA, Cudkowicz ME, Brown MV, et al. Biochemical alterations associated with ALS. Amyotroph Lateral Scler 2012;13:110-118.

27. Lawton KA, Brown MV, Alexander D, et al. Plasma metabolomic biomarker panel to distinguish patients with amyotrophic lateral sclerosis from disease mimics. Amyotroph Lateral Scler Frontotemporal Degener 2014;15:362-370.

28. Smith QR, Momma S, Aoyagi M, Rapoport SI. Kinetics of neutral amino acid transport across the blood-brain barrier. J Neurochem 1987;49:1651-1658.

29. Kanamori K, Ross BD, Kondrat RW. Rate of glutamate synthesis from leucine in rat brain measured in vivo by 15N NMR. J Neurochem 1998;70:1304-1315.

30. Van Damme P, Dewil M, Robberecht W, Van Den Bosch L. Excitotoxicity and amyotrophic lateral sclerosis. Neurodegener Dis 2005;2:147-159.

31. Contrusciere V, Paradisi S, Matteucci A, Malchiodi-Albedi F. Branched-chain amino acids induce neurotoxicity in rat cortical cultures. Neurotox Res 2010;17:392-398.

32. Kiernan MC, Vucic S, Cheah BC, et al. Amyotrophic lateral sclerosis. Lancet 2011, 377:942-955.

33. Stickler DE, Royer JA, Hardin JW. Accuracy and usefulness of ICD-10 death certificate coding for the identification of patients with ALS: results from the South Carolina ALS Surveillance Pilot Project. Amyotroph Lateral Scler 2012;13: 69-73.

34. Kioumourtzoglou MA, Seals RM, Himmerslev L, Gredal O, Hansen J, Weisskopf MG. Comparison of diagnoses of amyotrophic lateral sclerosis by use of death certificates and hospital discharge data in the Danish population. Amyotroph Lateral Scler Frontotemporal Degener 2015;16:224-229.

35. Townsend MK, Clish CB, Kraft P, et al. Reproducibility of metabolomic profiles among men and women in 2 large cohort studies. Clin Chem 2013;59:1657-1667. 


\section{Neurology}

\section{Prediagnostic plasma branched-chain amino acids and the risk of amyotrophic lateral sclerosis}

Kjetil Bjornevik, Éilis J. O'Reilly, James D. Berry, et al.

Neurology 2019;92;e2081-e2088 Published Online before print November 14, 2018

DOI 10.1212/WNL.0000000000006669

This information is current as of November 14, 2018

Updated Information \& Services

References

Subspecialty Collections

Permissions \& Licensing

Reprints including high resolution figures, can be found at: http://n.neurology.org/content/92/18/e2081.full

This article cites 34 articles, 9 of which you can access for free at: http://n.neurology.org/content/92/18/e2081.full\#ref-list-1

This article, along with others on similar topics, appears in the following collection(s):

All CBMRT/Null Hypothesis

http://n.neurology.org/cgi/collection/all_cbmrt_null_hypothesis Amyotrophic lateral sclerosis

http://n.neurology.org/cgi/collection/amyotrophic_lateral_sclerosis_ Cohort studies

http://n.neurology.org/cgi/collection/cohort_studies

Risk factors in epidemiology

http://n.neurology.org/cgi/collection/risk_factors_in_epidemiology

Information about reproducing this article in parts (figures,tables) or in its entirety can be found online at:

http://www.neurology.org/about/about_the_journal\#permissions

Information about ordering reprints can be found online:

http://n.neurology.org/subscribers/advertise

Neurology ${ }^{\circledR}$ is the official journal of the American Academy of Neurology. Published continuously since 1951, it is now a weekly with 48 issues per year. Copyright (O 2018 American Academy of Neurology. All rights reserved. Print ISSN: 0028-3878. Online ISSN: 1526-632X.

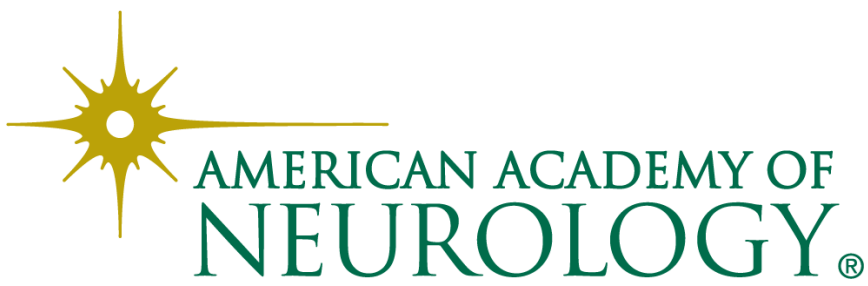

